ALÉTHEIA

Enero - Diciembre. 2019, Vol. N7, pp. 25-29

DOI: 10.33539/aletheia.2019.n7.2149

\title{
LOS TRASTORNOS DE APRENDIZAJE Y EL IMPACTO DE LAS NUEVAS TECNOLOGÍAS
}

\author{
THE LEARNING DISORDERS AND THE \\ IMPACT OF THE NEW TECHNOLOGIES
}

Sheila Mariza Elias Omarji de Alvarado

eliassyrio@yahoo.com.br

Universidad Femenina del Sagrado Corazón

\section{INTRODUCCIÓN}

Los trastornos de aprendizaje son trastornos de neurodesarrollo que afectan al 10\% de la población en edad escolar. Al respecto, se considera que tales trastornos "Son un término genérico que designa un conjunto heterogéneo de perturbaciones que se manifiestan por dificultades persistentes en la adquisición y en la utilización de la escucha, de la palabra, de la lectura, de la escritura, del razonamiento, de las matemáticas, o de habilidades sociales. Estos desórdenes son intrínsecos a la persona y son presuntamente causados por un disfuncionamiento del sistema nervioso central". Citado por J. P. Brunet (3: 1998).

Estos trastornos no son considerados como una enfermedad, en la mayoría de los casos los individuos afectados nacieron con esta condición. El origen de estos trastornos se da por causas neuronales del cerebro, por lo tanto, no tienen cura. El tratamiento diferenciado además del apoyo necesario en sus hogares es la clave para aprender a convivir con esta condición.

Los trastornos de aprendizaje más comunes son los siguientes: disorders and because of the inappropriate use of the new technologies the children and teenagers are presenting learning problems usually to be confused with learning disorders, and this abuse of new technologies can become addiction until develops mental illness.

Key words: Learning disorders, new technologies, video games, digital dementia, addiction.

\section{TIPOS}

\begin{tabular}{ll}
\hline \multicolumn{1}{c}{ TIPOS } & \multicolumn{1}{c}{ ÁREA AFECTADAS } \\
\hline a. Dislexia & Adquisición de la lectura \\
b. Digrafia & Calidad de la escritura \\
c. Disortografía & Expresión escrita \\
d. Disfasia & Aprendizaje del lenguaje oral
\end{tabular}




\section{e. Discalculia Aprendizaje de las matemáticas \\ f. Disfemia Ritmo, fluidez y melodía en el habla}

En la actualidad, por causa de la introducción de las nuevas tecnologías de comunicación e información en nuestra sociedad, en nuestras casas, en nuestros trabajos, etc, se está creando problemas conductuales y de aprendizaje muy severos en nuestros niños. Niños que nacieron sin ningún tipo de trastorno, y que por causa del uso indiscriminado y sin ningún tipo de control de estas nuevas tecnologías, están presentando problemas de aprendizaje a punto de tener características de los trastornos mencionados arriba. Hay que entender claramente que son los trastornos, su origen, y las consecuencias del impacto de las nuevas tecnologías, para que el maestro y los padres de familia puedan saber cómo intervenir para mejorar el proceso de enseñanza y aprendizaje y criar individuos que sean de provecho para la sociedad y no lo contrario.

\section{Tipo de trastornos Asociados}

\subsection{Trastorno de déficit de atención con hiperac- tividad - TDAH}

El TDAH, trastorno por déficit de atención e hiperactividad ha sido uno de los principales motivos de consulta en neuropediatría, aunque los criterios clínicos están bien establecidos, probablemente no se repare de manera rigurosa en la historia clínica y no se adviertan adecuadamente los síntomas más determinantes. (RN 2008)

Según Pérez et al. (2007) en el primer consenso latinoamericano de trastorno por déficit de atención y hiperactividad desarrollado en México, el TDAH, se define como alteraciones neurológicas del ser humano cuyos síntomas son inatención y problemas conductuales como impulsividad e hiperactividad; es un trastorno neuropsiquiátrico más común a nivel pediátrico. A nivel de Latinoamérica, ocupando entre 3 a $7 \%$ de la población.

El diagnóstico más eficaz del TDAH son observaciones; entrevistas; cuestionarios para los padres, profesores o las personas que están más tiempo con el niño; para evaluación del comportamiento si es variable o constante con los diversos grupos.

Las causas, mayoritariamente se tiene el factor hereditario, aunque otros factores influencian, como el componente del medio ambiente, la alimentación, hábitos de fumar de la madre, bajo peso al nacer, niños prematuros, alcoholismo, consumo de drogas, etc.

Tratamiento de TDAH, el más común es el uso de farmacológicos y las terapias alternativas.

\subsection{Trastornos de lenguaje}

Según Roseli (2010), Brocca en el siglo XIX empezó sus estudios que se extendieron en el siglo $\mathrm{XX}$, en especial en estudios del cerebro del adulto, se acercó a los estudios de lesiones cerebrales en niños y sus disfunciones congénitas, al final del siglo XX donde quedó más claro que los problemas de lenguaje y las afasias (Brocca y de Wernicke), son mayormente de origen congénito

\subsection{Trastorno de Aprendizaje no Verbal - TANV}

El trastorno del aprendizaje no verbal (TANV) es un grupo complejo de dificultades con sustrato biológico definido. Sus manifestaciones clínicas afectan al área motora, a la organización visual espacial y a las competencias sociales. (RN 2008).

\section{- Objetivos}

El objetivo de este artículo es llamar la atención a nuestra sociedad en general para controlar y supervisar el uso adecuado de estos aparatos inteligentes en posesión de nuestros hijos y evitar problemas psicológicos más graves.

\section{- Justificación}

La justificación de este artículo es para prevenir la proliferación de individuos que no sumen a la sociedad con un aporte positivo, y también para prevenir suicidios, por exceso de consumo de alcohol y de 
substancias químicas que afecten el funcionamiento normal del cuerpo humano por causa del uso y abuso indiscriminado de aparatos inteligentes.

"Estos problemas de aprendizaje no tienen origen en el cerebro, más bien en el uso excesivo de las nuevas tecnologías de la información. Los estímulos exteriores son los que provocan problemas para el progreso de enseñanza" (Roseli Et al 2010).

2. Características de las nuevas tecnologías de la información y comunicación (NTIC)

- Procesar datos de manera rápida y fiable;

- Automatizar tareas;

- Almacenar grandes cantidades de información;

- Establecer comunicaciones inmediatas, sincróni cas y asincrónicas,

- Trabajar y aprender colaborativamente;

- Producir contenidos y publicarlos en la web;

- Participar en comunidades virtuales.

\subsection{Efectos adversos de las nuevas tecnologías de la información y comunicación (NTIC) en el aprendizaje}

a. Los niños utilizan cada vez más las NTIC, y pueden volverse adictos, convirtiéndose además en un problema de lenguaje por su falta de comunicación con los demás.

b. Agresión (los juegos violentos); los videojuegos agresivos, terminan volviendo a los niños, más agresivos, a punto de idealizar los juegos como su vida real.

c. Acceso a sitios web (a veces dañinos), chateando en línea en facebook, compartiendo fotos en whatsapp, instagram, snapchat, etc; con personas desconocidas y algunos con intenciones perversas.

d. Disminución de la calidad y la cantidad de sueño. La melatonina química del sueño está siendo influenciada por el brillo constante de las pantallas de estas NTIC; e. Estrés crónico de los teléfonos inteligentes. Es causado por notificaciones (o su ausencia), anticipación constante de un mensaje o correo electrónico. Si una persona no recibe los mensajes suficientes.

f. Problemas de salud. Psicológicos (conducta, irritabilidad, ansiedad, estrés, etc), de visión (ojos secos), de audición, metabólicos como la obesidad, etc.

\subsection{Demencia digital}

El doctor Manfred Spitzer, psiquiatra y neurocientífico, ha centrado su carrera en el estudio del cerebro y lleva años alertando sobre las nuevas tecnologías. Sobre la introducción de computadores portátiles en primaria, dice lo siguiente:

"No se trata de otra cosa que de una especie de enganche. Tenemos el ejemplo de Corea del Sur, donde su gobierno reconoció el 2010 que ya entonces el $12 \%$ de todos los escolares eran adictos a internet".

Precisamente, el término "demencia digital" procede de este país. La adicción por los videojuegos es un problema mental.

El doctor Pedro Horvat, psiquiatra y psicoanalista explica lo siguiente:

La esencia de los juegos es premiar la permanencia y el esfuerzo, dando recompensas en forma variable. Un mecanismo propio de -por ejemplo- las máquinas tragamonedas, que incita la compulsión y genera adicción en las personas más predispuestas. En pruebas de laboratorio con ratones, al enfrentarlos con una palanca que al usarse libera comida en cantidades aleatorias, la respuesta es la misma: la usan compulsivamente. Los videojuegos no son la excepción. Los escenarios que plantean facilitan la inmersión en un mundo de fantasía. El usuario se identifica con situaciones y personajes a la vez que se van desconectando de la realidad. La música que se repite en loop y los efectos sonoros contribuyen al efecto hipnótico.

La respuesta neuroquímica y psicológica son equivalentes a otros tipos de conductas que se terminan 
transformando en adicciones. Está comprobada la sensación placentera por la liberación de dopamina en el cerebro y la reducción de ansiedad ante la posibilidad de jugar. Ambas respuestas están presentes en todas las adicciones.

Este problema se caracteriza por un patrón de comportamiento de juego "continuo o recurrente". La OMS lo vincula a tres condiciones negativas producidas por el abuso de los juegos digitales, que son las siguientes:

$1^{\circ}$ - Pérdida de control de la conducta en cuanto al inicio, frecuencia, intensidad, duración, finalización $\mathrm{y}$ contexto en que se juega.

$2^{\circ}$ - Produce un aumento de la prioridad que se otorga a los juegos frente a otros intereses vitales y actividades diarias.

$3^{\circ}$ - Relación con la escalada o mantención de la conducta, pese a la ocurrencia de las otras dos consecuencias negativas.

Una institución médica llamada reSTART, se convirtió en el primer retiro terapéutico en Estados Unidos dedicado específicamente a la adicción de adolescentes a Internet después de recibir una avalancha de llamadas de padres que estaban desesperados por separar a sus hijos de los videojuegos, las computadoras y los teléfonos celulares. Un pequeño pero creciente número de psicólogos en Estados Unidos comenzó a especializarse en el tratamiento de niños que luchan contra el uso compulsivo de las NTIC.

\subsubsection{Signos y síntomas de la adicción a los vi- deojuegos}

- Superar las 25-30 horas de juego semanal;

- Necesidad irresistible de jugar y/o respuestas emocionales intensas cuando esto no es posible;

- Debilitamiento del rendimiento escolar o laboral por el juego;

- Marcadas expresiones de ansiedad y/o euforia durante la actividad;
- Empobrecimiento de los vínculos sociales y de los intereses en general fuera del juego;

- Dificultades para dormir u otros cambios de hábitos significativos.

\section{Discusión}

El uso de las nuevas tecnologías de información y comunicación (NTIC), tiene evidentemente muchos aspectos positivos y algunos aspectos negativos relevantes que afectan especialmente a nuestros hijos. El uso de aparatos inteligentes y videojuegos está creando una adicción a niveles insospechados en esta nueva generación. Se está concretizando el pensamiento de Albert Einstein cuando decía: "Temo el día en que la tecnología sobrepase nuestra humanidad; el mundo solo tendrá una generación de idiotas".

\section{CONCLUSIONES}

Una serie de estudios han demostrado que los niños que gastan largas horas usando las nuevas tecnologías, tienen mayor riesgo de desarrollar problemas de aprendizaje que pueden ser diagnosticados como trastornos de aprendizaje. El uso de las nuevas tecnologías trae frecuentemente un cambio radical en el comportamiento del niño, desde su conducta (irritabilidad, hiperactividad, estrés, ansiedad), en su metabolismo (obesidad), en el lenguaje, insomnios, pérdida de memoria, pérdida de visión y audición, y en su capacidad de aprender. La adicción a los videojuegos provoca que el cerebro se vaya habituando a niveles altos de neurotransmisores (dopamina, serotonina) en ciertas áreas y el cerebro cada vez pide más para repetir la acción. Este desequilibrio entre neurotransmisores excitadores e inhibidores a nivel cerebral puede ser causa de enfermedades mentales futuras. 


\section{REFERENCIAS}

Bravo-Valdivieso, L., Milicic-Müller, N., Cuadro, A., Mejía, L., Eslava. (2016). Revista SciELO Analytics. Artículo “Trastornos del Aprendizaje: Investigaciones Psicológicas y Psicopedagógicas en diversos países de Sud América”, Ciencias Psicológicas. Versión On-line ISSN 1688-4221, Cienc. Psicol. Vol. $3 \mathrm{~N}^{0} 2$ Montevideo, nov. 2009

Bravo-Valdivieso, L.; Milicic-Müller N.; Cuadro, A.; Mejía L.; Eslava (2016). Revista SciELO Analytics. Artículo "Trastornos del Aprendizaje: Investigaciones Psicologicas y Psicopedagogicas en diversos países de Sud America”, Ciencias Psicológicas versión On-line ISSN 1688-4221, Cienc. Psicol. vol.3 no.2 Montevideo nov. 2009.

Denzin NK. Triangulation in Educational Research. En: Keeves JP, editor. Educational Research, Methodology and Measurement: An International Handbook. Londres: Pergamon; 1997. p.318- 321.

Dussel I. Aprender y enseñar en la cultura digital. Buenos Aires: Santillana; 2011.

E. Barragán Pérez, F. de la Peña-Olvera, S. Ortiz-León, M. Ruiz-García, J. Hernández-Aguilar, L. Palacios-Cruz, A. Suárez-Reynaga. Artículo: "Primer consenso latinoamericano de trastorno por déficit de atención e hiperactividad desarrollado", Recuperado de: http://tdahlatinoamerica.org/ documentos/05_CARPETA_5_Barragan__ y_otros Primer_Consenso.pdf

Fung C, Kuhle S, Lu C, Purcell M, Schwartz M, Storey K, Veugelers PJ. From "best practice" to "next practice": the effectiveness of school-based health promotion in improving healthy eating and physical activity and preventing childhood obesity. International Journal of Behavioral Nutrition and Physical Activity. 2012; 1-9. Disponible en: http://www. ijbnpa. org/content/9/1/27

Goikoetxea, E. (2012). Las dificultades específicas del aprendizaje en el albor del siglo xxi.relieve. Revista Electrónica de Investigación y Evaluación Educativa, 18 (1), 1-16.

Horvat P., Psiquiatra. "La OMS reconoce al trastorno por videojuegos como un problema mental" http://www.somosnext.net/en/novedad/ la-organizacion-mundial-de-la-salud-reconoce-adiccion-a-videojuegos-como-un-problema-mental-1

Quintero J, Munévar RA, Munévar FI. Nuevas tecnologías, nuevas enfermedades en los entornos educativos. Hacia promoc. salud. 2015; 20(2): 13-26. DOI: 10.17151/hpsal.2015.20.2.2

Roseli, M., Matute, E., Ardila, A. (2010). Neuropsicología del desarrollo infantil. México: Editorial Manuel Moderno

Rueda R. De los nuevos entramados tecnosociales: emergencias politicas y educativas. Folios. 2011; 33: 7-22.

Spitzer M., "Demencia Digital. El peligro de las nuevas tecnologías" Ediciones B, Barcelona, 2013, 373

Prensky M. On the Horizon. MCB University Press. 2001; 9(5).

Prensky M. The emerging online life of the digital native. 2004. Disponible en: http://www.marcprensky.com/writing/.
Fecha de Recepción: 12 de Agosto de 2019

Fecha de Aceptación: 27 de Noviembre de 2019 